\title{
Quantifying Skin Stretch induced Motion Artifact from an Electrocardiogram signal-A Pilot Study
}

Kalra A*, Lowe A, Al-Jumaily AM

Institute of Biomedical Technologies, Auckland University of Technology, New Zealand

\begin{abstract}
This work presents a 2D quantification of strain field caused due to the motion artifact in an Electrocardiogram (ECG) measurement. The objective of this work is to estimate the skin stretch induced motion artifact in an ECG signal. An ECG measurement was obtained from a subject for 10 seconds using standard $\mathrm{Ag} / \mathrm{AgCl}$ electrodes by continuously moving the arm back and forth during the measurement. A Poly dimethyl siloxane (PDMS) patch of dimensions $40 \mathrm{~mm} \times 45 \mathrm{~mm} \times 0.254 \mathrm{~mm}$ was adhered to the arm during motion. The movement of the PDMS patch during the ECG measurement was recorded in a video and motion artifact was quantified in terms of normal and shear strain components $\varepsilon x, \varepsilon y$ and $\varepsilon x y$. These values were derived using feature detection and Euclidean distance feature mapping. The motion artifact was eliminated from the ECG signal using Extended Kalman Filtering (EKF).
\end{abstract}

Keywords: Skin stretch; 2D quantification; Motion artifact; Electrocardiogram signal; Poly dimethyl siloxane

\section{Introduction}

Electrocardiogram or ECG is an important clinical diagnostic measure which is widely known and practiced. Principally, it reflects the activity of the heart and cardiovascular metabolism occurring inside a living being in the form of electrical impulses over time. Usually, it is used to diagnose conditions of Cardiac Arrhythmias, murmurs, Pulmonary Embolism etc. [1]. For example, a 12 lead ECG shows alterations that may include left ventricular hypertrophy, and increased QRS voltages in cases of athletic populations. Ambulatory ECG biosensors aim for patient comfort and ease of use with efficient signal acquisition. This targets the infrequent cardiac arrhythmias which normally pass undetected in clinical situations. The ambulatory ECG biosensor provides continuous monitoring of the heart for 2448 hours while the patient is performing his or her daily activities. Artifacts induced in an ambulatory ECG include noise from the outside environment. This noise interferes with the low voltage ECG signal, thereby resulting in distortion in the obtained ECG pattern. Artifacts might occur due to the movement of electrodes on the surface of the skin and power line interference [2]. These artifacts can be reduced to a great extent using instrumentation amplifier and band pass filters [3]. Although many ambulatory ECG monitoring biosensors have been commercialised to date, a major problem is still faced due to patients performing motion related activities that introduce unwanted signal noise and makes monitoring less effective [4]. Various motion biosensors used to date to remove motion related noise don't respond well in cases where the patient is performing vigorous exercises. In [5], the motion artifact has been evaluated in terms of displacement using optical sensors. The overall performance of these motion sensors accounted for $85 \%$ reduction in motion artifact. The key challenge faced in implementation of an ambulatory ECG system is the deterioration of signal due to movement of the patient, which results in skin stretch, causing a change in position of the electrode with respect to skin.

Skin stretch has been identified as one of the major factors contributing to motion artifact [5]. Skin can be considered as a current generator which generates a potential difference of around $30 \mathrm{mv}$ between the inside and the outside of the skin. Stretching of skin can considerably reduce this potential difference by $5 \mathrm{mv}$, which introduces motion artifact [6]. Other factors, such as electrode-skin impedance and potentials created due to gel movement in-between the electrode/ skin interface have a negligible effect on the disturbance produced in the ECG pattern.

This paper investigates the use of the non-uniform strain field pertaining to skin stretch of the patient to reduce ECG motion artifact. This differs from the existing approaches as they have been limited to the use uniaxial displacement [5], sensor acceleration [7] or other sources which do not directly measure or address skin stretch as a primary cause of motion artifact.

\section{Materials and Methods}

An ECG measurement was taken from a subject for 10 seconds using PhysioFlow ${ }^{\circledR}$ (Manatec Biomedical, Paris, France) through a National Instruments Data Acquisition board (NI-DAQ, Austin TX, USA). Standard $\mathrm{Ag} / \mathrm{AgCl}$ electrodes were placed close to the radial artery on the dorsal sides of both forearms as shown in Figure 1a. The left forearm of the subject with ECG lead I was continuously moved back and forth during the measurement, while the other forearm with ECG leads II was kept stable. A poly-dimethyl-siloxane (PDMS) patch of dimensions $40 \mathrm{~mm} \times 45 \mathrm{~mm} \times 0.254 \mathrm{~mm}$ with similar elasticity to skin was adhered to the arm in vicinity of the ECG electrode using polyvinyl alcohol (PVA) adhesive. The movement of the patch during the ECG measurement was recorded using a video camera with resolution 1920 pixels $\times 1080$ pixels at 25 frames per second. The PDMS patch has a checkerboard pattern printed on it to identify the corner points of the checker boxes. All the frames were filtered, sharpened and cropped in Photoshop CC (Adobe, San Jose, CA, USA). Corner points were identified using the Harris-Stephens algorithm in MATLAB (Mathworks, Natick, MA, USA), and track their movement over consequtive frames as shown in Figures $1 \mathrm{~b}$ and $1 \mathrm{c}$. The Euclidean

*Corresponding author: Anubha Kalra, Institute of Biomedical Technologies, Auckland University of Technology, New Zealand, Tel: 6499219999; E-mail anubha.kalra@aut.ac.nz

Received March 30, 2016; Accepted April 20, 2016; Published April 30, 2016

Citation: Kalra A, Lowe A, Al-Jumaily AM (2016) Quantifying Skin Stretch induced Motion Artifact from an Electrocardiogram signal-A Pilot Study. J Biosens Bioelectron 7: 204. doi:10.4172/2155-6210.1000204

Copyright: ( 2016 Kalra A, et al. This is an open-access article distributed under the terms of the Creative Commons Attribution License, which permits unrestricted use, distribution, and reproduction in any medium, provided the original author and source are credited. 
distance method was applied to match points from the initial frame to the next frame by considering the minimum projected distance between them. This was achieved by choosing the least distant to be a mapped pair.

The main goal of this research is to track skin stretch under the ECG electrode as a 2-dimensional strain field in order to improve the Signal to Noise ratio (SNR) of an ECG signal. The movement of the patch was quantified in terms of normal and shear strain components $\varepsilon x, \varepsilon y$ and $\varepsilon x y$ by using the finite strain theory [8].

A step-by-step approach demonstrating the quantification of motion artifact from an ECG signal is given in Figure 2.

The strain values were computed from the displacements of corner points of the checkerboard boxes for all the video frames. Delaunay triangulation calculation was used to obtain triangles from the elements undergoing deformation with respect to the first frame. The normal and shear strains calculated from the finite strain theory equation in two dimensions (2D) were mapped at the centroids of the triangles. Mean values of $\varepsilon x, \varepsilon y$ and $\varepsilon x y$ were calculated over the entire range of image frames. The maximum principal strain was calculated from these strain components and was used to reduce motion artifact from the noisy ECG signal through Extended Kalman Filtering.

\section{Results and Discussion}

The recorded motion signal was processed using image processing and point tracking. The motion artifact was calculated using finite strain equations as discussed above and can be seen in Figure 3. The video was recorded at 10 seconds at 25 frames per second. It can be observed from Figure 3 that the range of strain values lies within -0.02 to 0.07 , which is a plausible range of strain for skin under these conditions. Signal fluctuations corresponding to the pseudo-periodic motion of the arm is also observed (Figure 3).

The vector plots representing the change in position of the checkerboard corners of the corresponding frame from the first frame of reference can be seen for frames 55, 56 and 57 in Figure 4. The direction of the arrows represents the direction in which the points have moved. The strain vectors $\varepsilon x$, ey and exy evaluated from the vector points are mapped on incentres of the Delaunay triangles for each frame as discussed above. Strain contour plots of the respective frames corresponding to the vector plots are shown in Figures $5 \mathrm{a}, 5 \mathrm{~b}$ and $5 \mathrm{c}$.

The recorded ECG signal comprises of the true ECG signal, environmental noise and the motion artifact. The environmental noise and the noise due to interference with the mains power line can be filtered by using an instrumentation amplifier and a band pass filter. In this case, hardware filtering was provided by the PhysioFlow.

ECG measurements acquired from the subject with and without movement for 10 seconds are demonstrated in Figure 6. From Figure 6, it can be observed that the ECG voltage of the subject lies betwin $1 \mathrm{mv}$ to $1.8 \mathrm{mv}$. Under same environmental conditions, a significant change in the ECG pattern can be observed between Figure $6 \mathrm{a}$ and $6 \mathrm{~b}$ due to the effect of motion artifact.

Software processing of the collected raw signal thus becomes necessary to ensure a required quality of the signal within the required range (of frequency or time). A typical set of algorithms serves to remove noise and the motion artifacts, detect the heartbeats (QRS complex) and compress the data for efficient acquisition [9]. Although the important part for the ECG detection and conditioning is to obtain an accurate QRS pattern, the interest here is to detect the motion artifacts due to skin. Normal procedure for unwanted jitter and noise removal involves fixed frequency high pass and low pass filtering to concentrate the signal into the required bandwidth as seen in Figures $6 \mathrm{c}$ and $6 \mathrm{~d}$.

Extended Kalman Filter has been implemented to denoise the ECG signal. A set of state space equations to model ECG were developed as shown in equation 1.

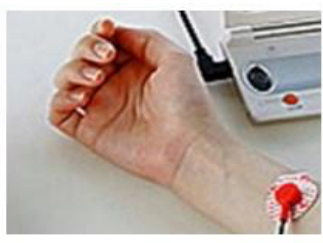

a

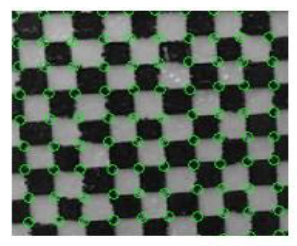

b

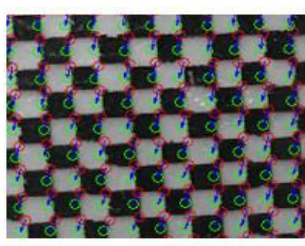

C

Figure 1: a. ECG measurement using PhysioFlow ${ }^{\circledR}$,1b. Checkerboard corner detection,1c. Point tracking.

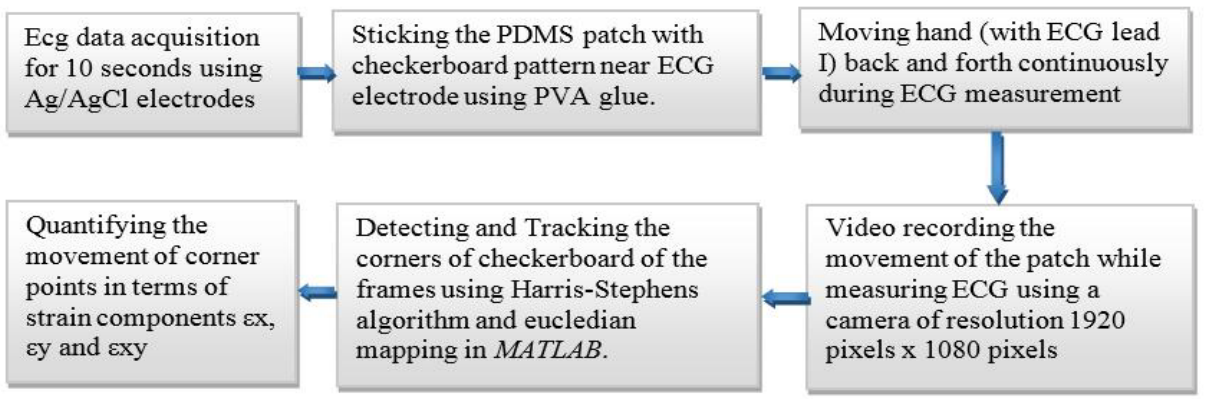

Figure 2: Block diagram representing the quantification of ECG motion artefact. 
Citation: Kalra A, Lowe A, Al-Jumaily AM (2016) Quantifying Skin Stretch induced Motion Artifact from an Electrocardiogram signal-A Pilot Study. J Biosens Bioelectron 7: 204. doi:10.4172/2155-6210.1000204

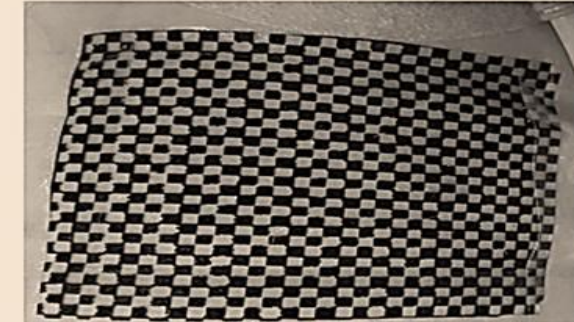

a. PDMS patch glued to skin

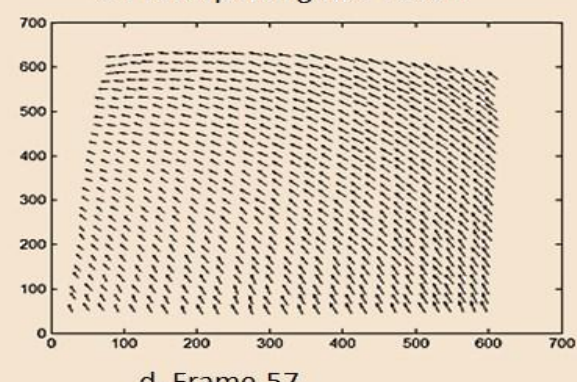

d. Frame 57
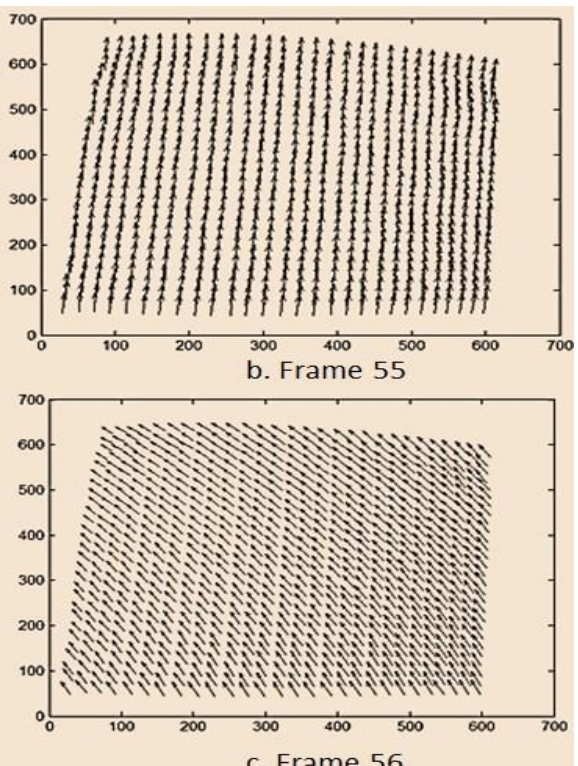

c. Frame 56

Figure 3: A plot of normal and shear strain components with time.

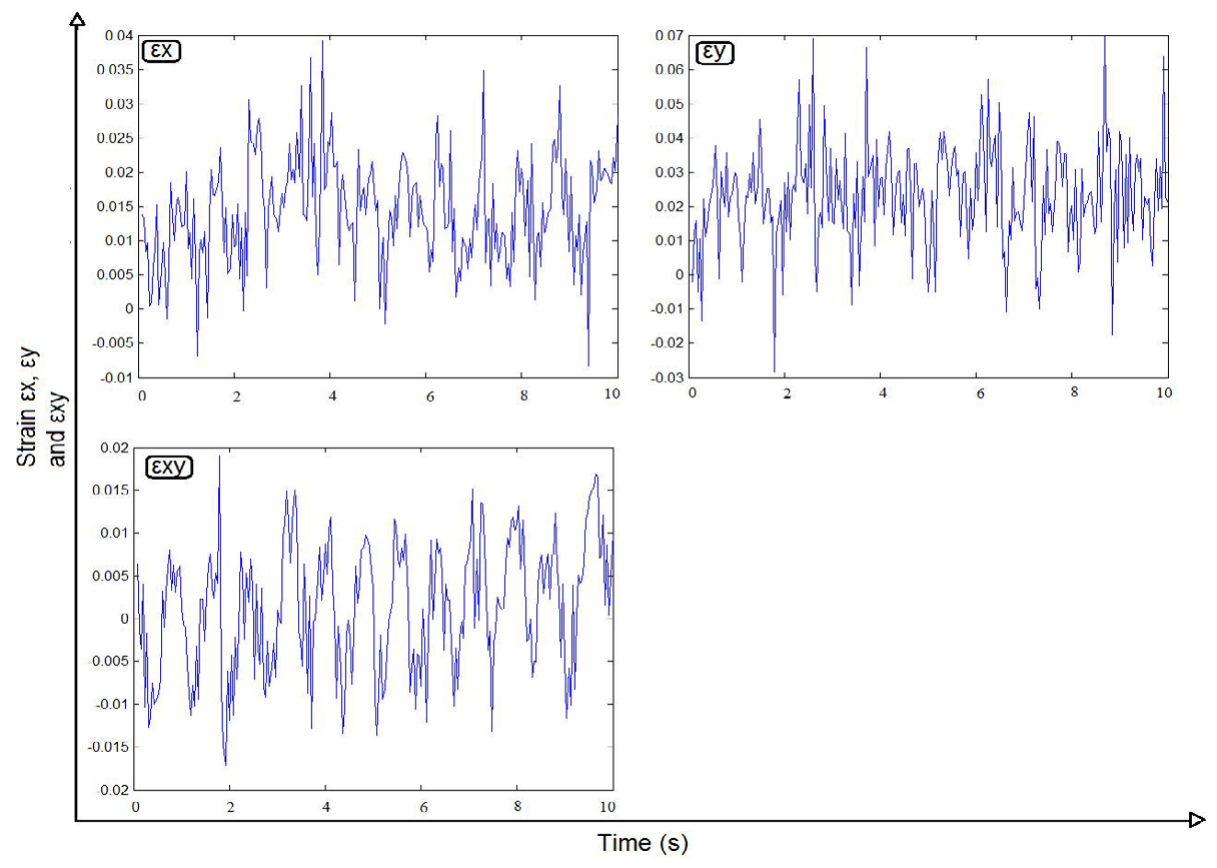

Figure 4: PDMS patch adhered to the arm and displacement vectors from the first frame.

$$
z_{k+1}=-\frac{\substack{\theta_{k+1}=\left(\theta_{k}+\delta\right) \bmod 2 \pi \\ \sum_{\mathrm{i}} \delta \mathrm{a}_{\mathrm{i}} \omega}}{b_{i}^{2}\left(\Delta \theta_{i} \exp \left(-\frac{\Delta \theta_{i}^{2}}{2 b^{2}}\right)\right)}+z_{k}+\eta
$$

Here $\mathrm{Zk}$ represents the ECG magnitude at time $\mathrm{k}$ at a sampling period $\delta, \Delta \theta \mathrm{i}=\theta \mathrm{k}-\theta \mathrm{i} \bmod (2 \pi)$ where $\theta$ is a phase variable and summation $i$ is taken over the number of guassian functions which can be used to get a desired ECG signal. Here summation $i$ is taken over $\mathrm{P}, \mathrm{Q}, \mathrm{R}, \mathrm{S}$ and $\mathrm{T}$ waves of the ECG signal and $\mathrm{\theta i}$ represents the guassian centre, ai denotes the guassian peak and $\beta \mathrm{i}$ represents the guassian width. The other parameters $\omega$ and $\eta$ represent the process and white guassian noise respectively. $\mathrm{Zk}$ and $\Theta \mathrm{k}$ hold for the state space equations of the Extended Kalman Filter. The dynamic ECG model is linearized continuously by updating the state equations with time. In an Extended Kalman Filtering approach, a priori information about the ECG dynamics is used to extract the ECG signal from the background noise. In this case, the strain values can be used as background noise or motion artifact information. Figure 7 shows ECG filtering using Extended Kalman Filtering where the maximum principal strain component (E1) evaluated from the ex, ey and exy values was used to reduce motion artifact from a noisy ECG signal (Figure 7). 
Citation: Kalra A, Lowe A, Al-Jumaily AM (2016) Quantifying Skin Stretch induced Motion Artifact from an Electrocardiogram signal-A Pilot Study. J Biosens Bioelectron 7: 204. doi:10.4172/2155-6210.1000204

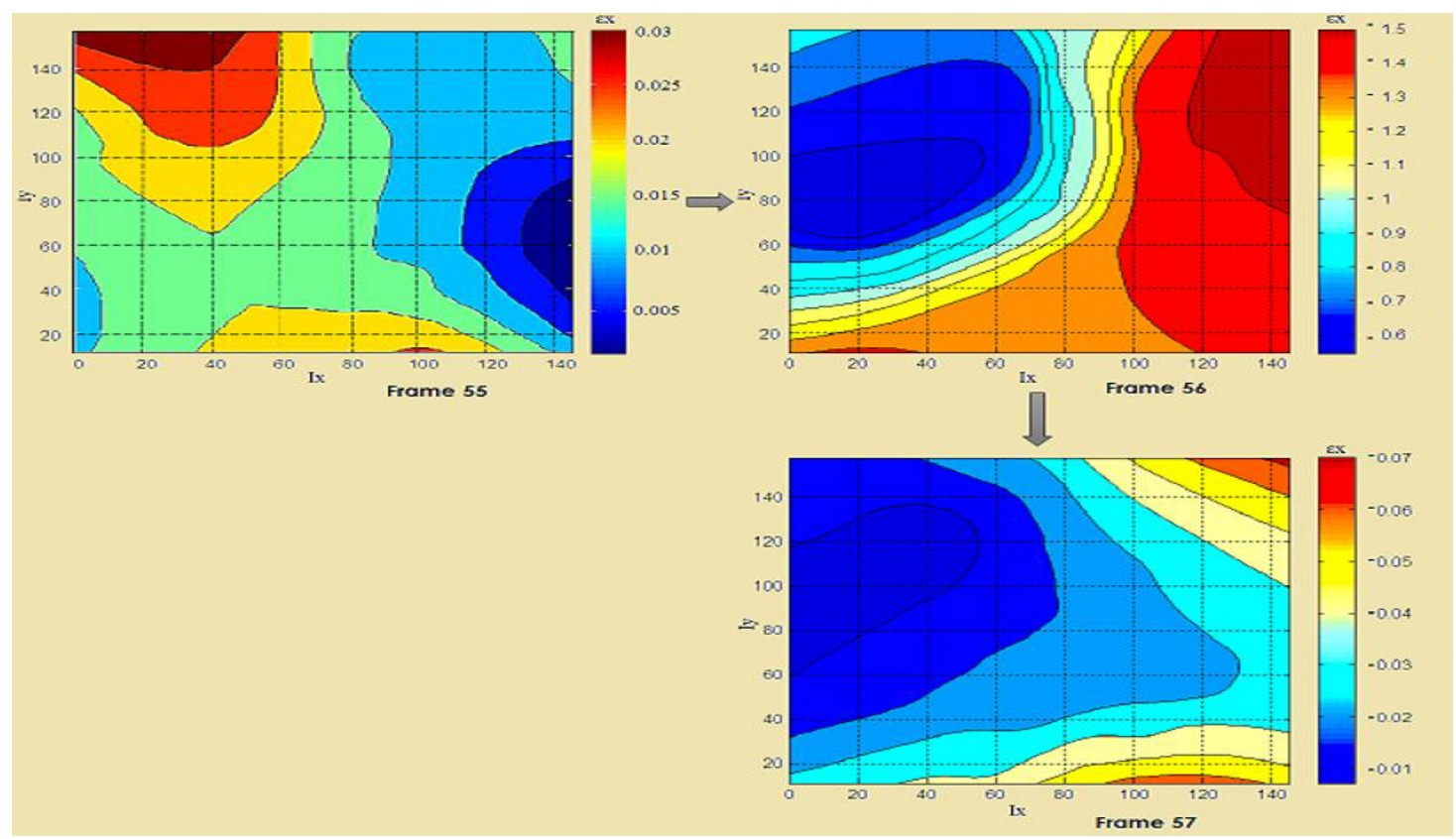

Figure 5a: Contour plots showing change in normal strainex with change in displacement.

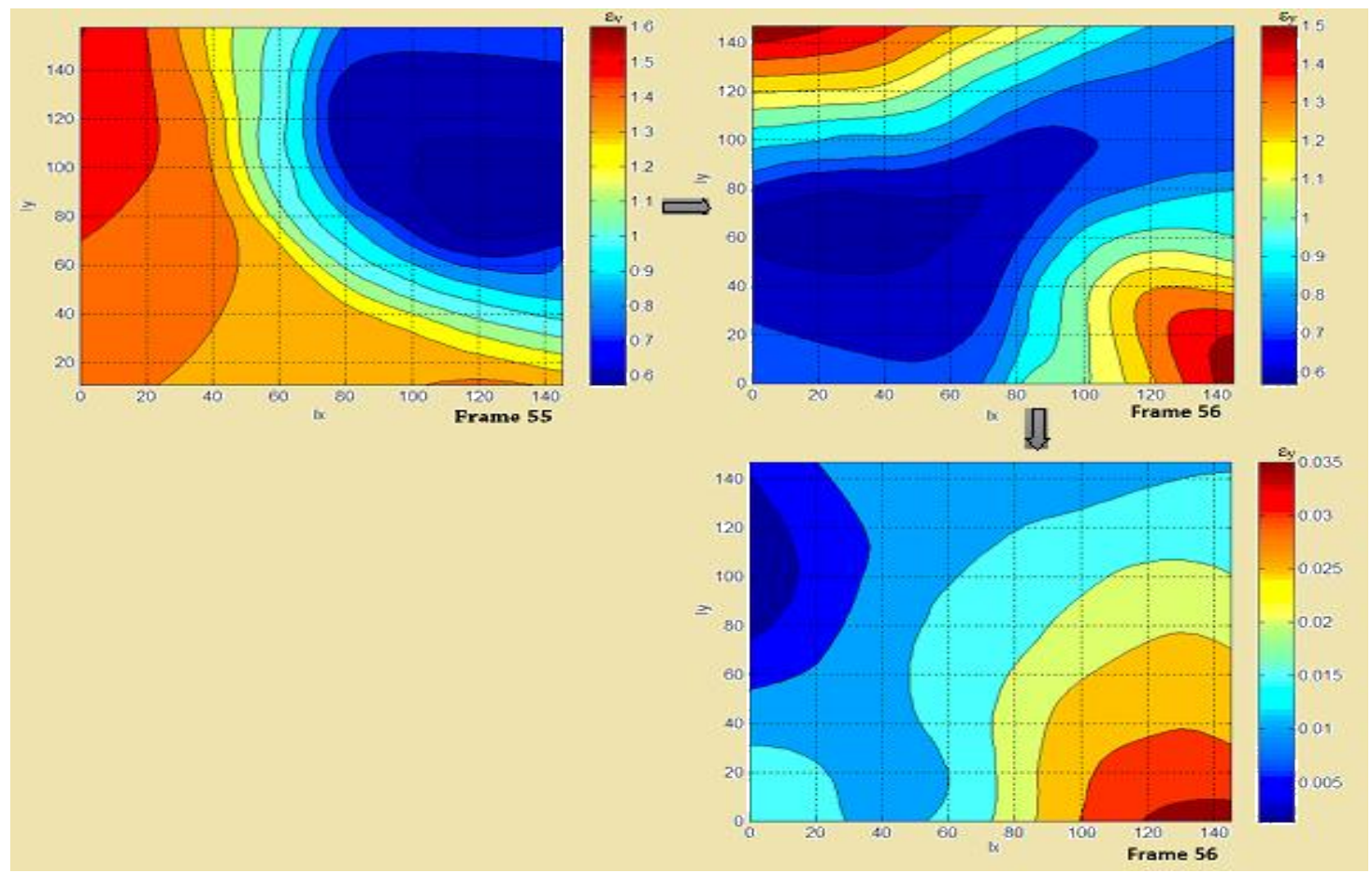

Figure 5b: Contour plots showing change in normal straincywith change in displacement.

\section{Future Work}

Other than Extended Kalman Filtering as implemented in this paper, several other techniques can be used for motion artifact elimination. The other techniques employed for modern signal processing are through wavelet transform and Independent Component Analysis (ICA). The wavelet transform is a proven tool for efficient filtering of signals and can prove much useful in bio-signal processing as well [10-12]. It involves the decomposition of the signal, followed by its thresholding and then the proper reconstruction [13]. One of the works [14] used different wavelets to reduce motion artifacts from corrupted PPG signals. The wavelets used included the Daubechies, biorthogonal, symlet, Coiflet etc. out of which the Daubechies produced the best performance. Even the amount of variety available through 
Citation: Kalra A, Lowe A, Al-Jumaily AM (2016) Quantifying Skin Stretch induced Motion Artifact from an Electrocardiogram signal-A Pilot Study. J Biosens Bioelectron 7: 204. doi:10.4172/2155-6210.1000204
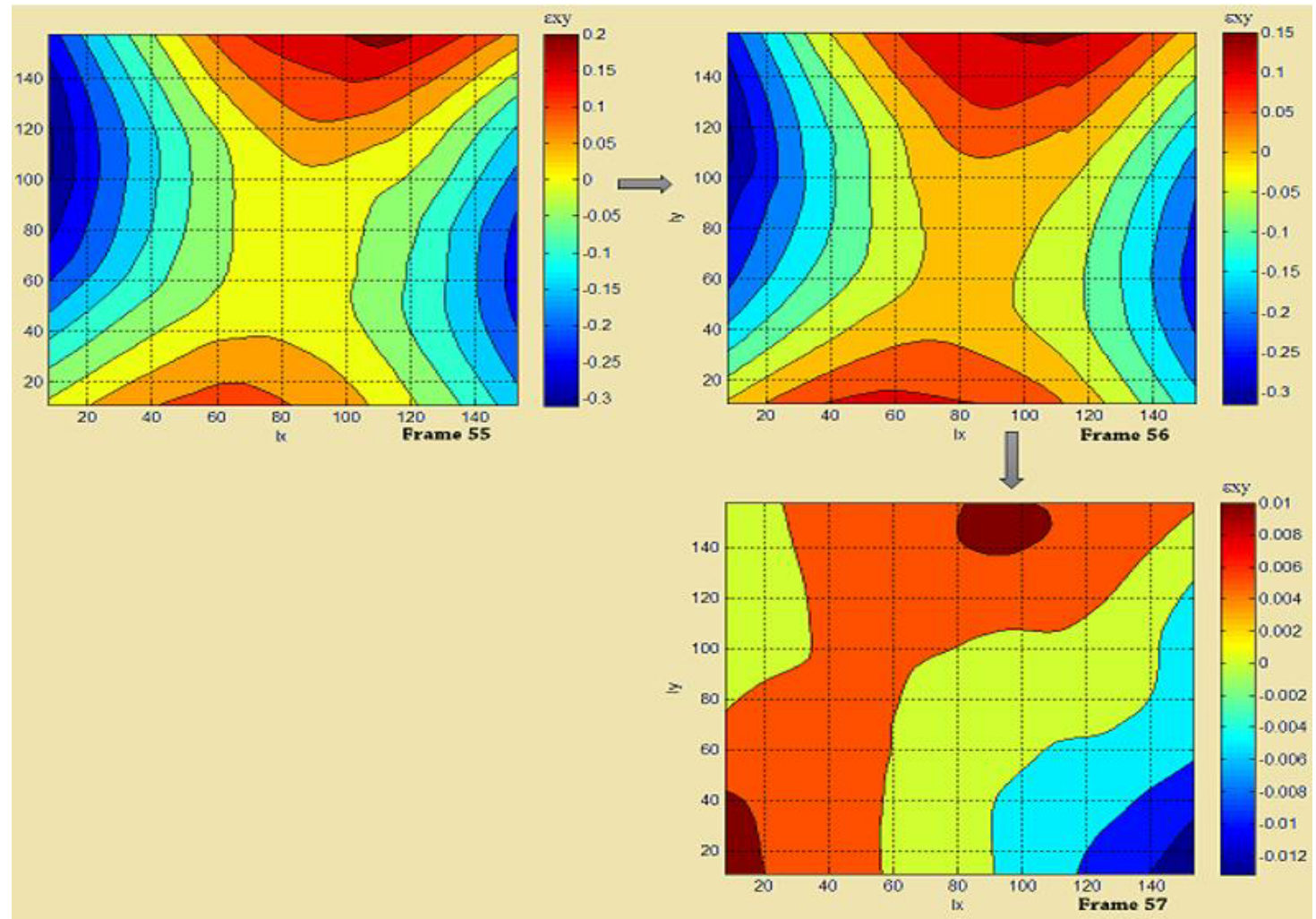

Figure 5c: Contour plots showing change in shear strainexy with change in displacement.

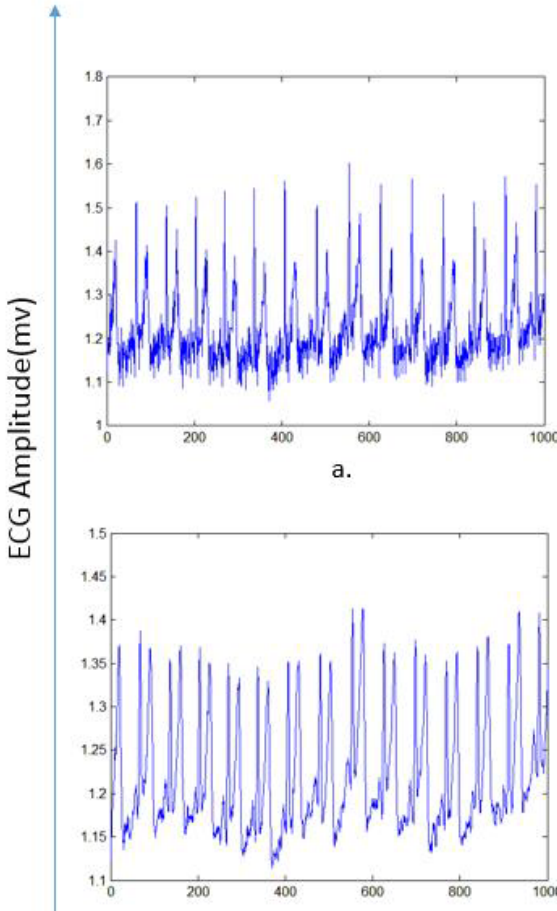

C.
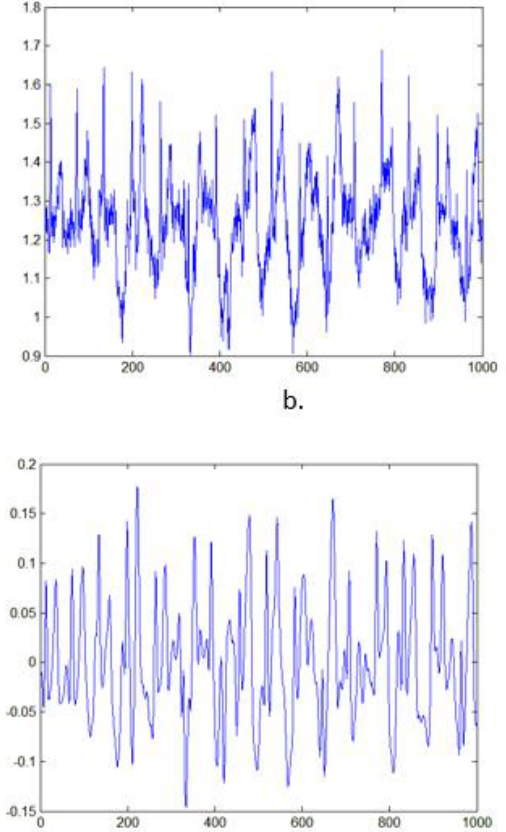

Frequency $(\mathrm{Hz})$

Figure 6: a. ECG with motion artifacts b. ECG without motion artifacts c. Filtering of ECG signal (a) d. Filtering of ECGsignal (b). 


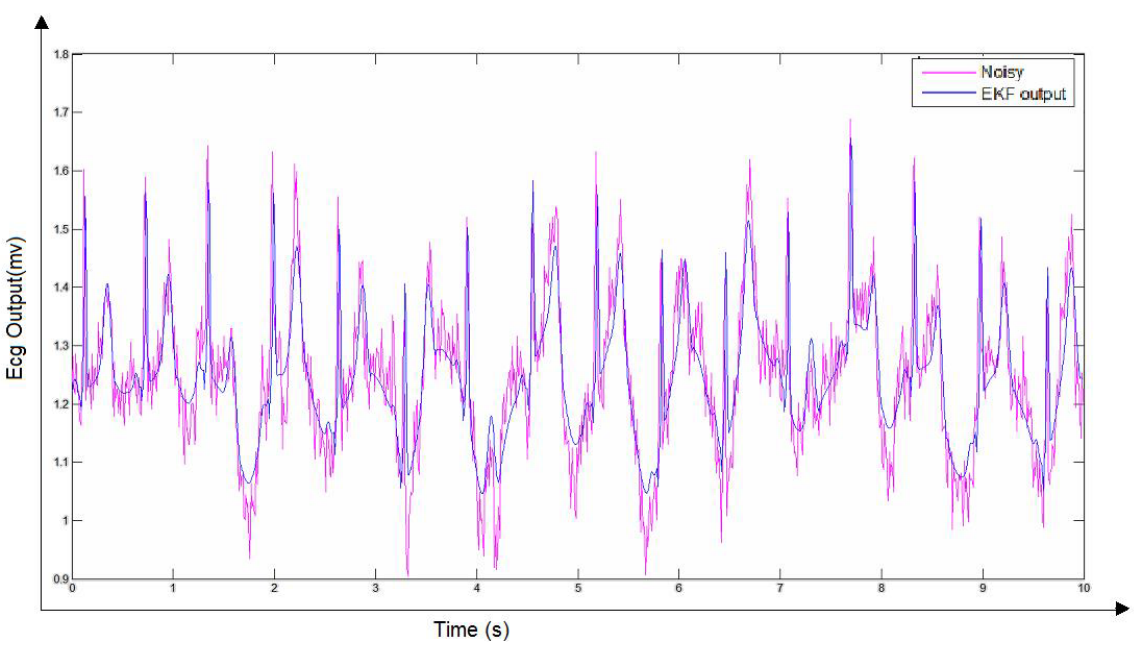

Figure 7: ECG with motion artifacts and filtered ECG using extended Kalman filter.

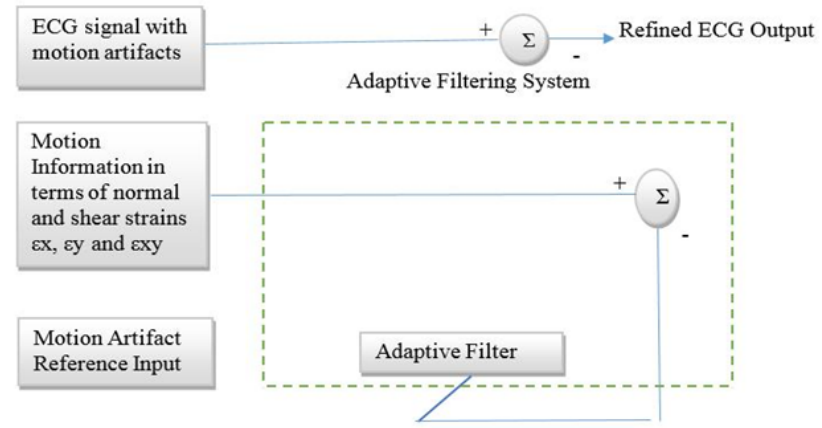

Figure 8: Signal processing using adaptive filtering.

different wavelets, the wavelet transform has a limited application which was pointed out in [15] through the analysis of corrupted PPG signals.

ICA separates the signal with multiple variants into components by assuming that the components are statistically independent [16-18]. For bio-signal such as ECG, ICA has been found to be increasingly used since it does not require any prior knowledge of the system $[19,20]$.

Adaptive filtering is also one of the most dominant filtering techniques, especially for the treatment of bio-signals [21]. Thakur and Zhu, applied it in fetal ECG recording, cancelling the cardiogenic interference signal from that obtained from Impedance Plethysmography (IPG), noise reduction from muscles, cancelling the $60 \mathrm{~Hz}$ power supply interference and ECG motion artifact reduction [22]. Many previous works have stated the use of adaptive techniques like adaptive neural networks [23] and fuzzy based adaptive non-linear filters [24] to check the ECG motion artifacts.

Generally, adaptive filtering is realized by the subtraction of the noise from the received signal in an adaptive manner. The noise essentially is the unwanted component, here being the skin motion artifacts. As could be understood, the technique employs two input architecture, one being the overall ECG signal, and the other being the noise source. As seen in Figure 8, the adaptive filter estimates the noise from its source sensor which is then subtracted from the first input [5].

\section{Conclusion}

This paper aims at introducing a novel technique for the removal of skin stretch induced motion artifacts from ECG signals. This approach to simulate the strain values reflecting skin stretch is accomplished by acquiring a video recording of a moving PDMS patch glued to the subject's arm during the ECG measurement. This was followed by the superposition of the strain vectors $\varepsilon x$, ey and exy pertaining to skin stretch. To account for deformation measurements, a predetermined checkerboard pattern was marked on the film to fulfil the best fit triangular geometry and to aid in motion tracking. Vector plots representing the displacements of corner points of the checkerboard from the first frame were obtained over the period of 10 seconds. A simple yet effective method of point tracking was employed and no corner point was lost while tracking. The quality of the frames was improved using Adobe Photoshop CC and the region of interest was cropped in MATLAB. Vector plots corresponding to displacement from the first frame were found to be in good agreement with the strain field contour mapping of their respective frames.

The objective of evaluating normal and shear strains was accomplished by mathematically relating them to the displacement of an infinitesimal particle due to skin stretch. A strain field pertaining to the movement of the arm was obtained over a period of 10 seconds. This was accounted for the motion information and was removed from the measured ECG using Extended Kalman Filter. 
Citation: Kalra A, Lowe A, Al-Jumaily AM (2016) Quantifying Skin Stretch induced Motion Artifact from an Electrocardiogram signal-A Pilot Study. J Biosens Bioelectron 7: 204. doi:10.4172/2155-6210.1000204

\section{References}

1. Bowden C, Masters J (2001) Veterinary nursing medical textbook. Oxford: Butterworth-Heinemann.

2. Government of New Zealand (2013) Financial statements of the Government of New Zealand for the year ended 30 June 2013, Financial Statements of the Government, New Zealand p: 186

3. ECG circuit analysis and design, Engineers labs

4. Vala D, Pawar T, Thakar VK (2007) Motion artifact removal in ambulatory ECG Signal using ICA

5. Liu $Y$ (2006) Reduction of skin stretch induced motion artifacts in electrocardiogram monitoring using adaptive filtering pp: 6045-6048.

6. Webster JG (1984) Reducing motion artifacts and interference in biopotential recording. IEEE Trans Biomed Eng 31: 823-826.

7. Raya MAD, Sison LG (2002) Adaptive noise cancelling of motion artifact in stress ECG signals using accelerometer. In Engineering in Medicine and Biology, 24th Annual Conference and the Annual Fall Meeting of the Biomedical Engineering Society EMBS/BMES Conference, Proceedings of the Second Joint 2: 1756-1757.

8. https://en.wikipedia.org/wiki/Finite_strain_theory

9. Sornmo L, Laguna $P$ (2006) Electrocardiogram (ECG) signal processing. Wiley Encycl Biomed Eng.

10. Chui CK (1992) An introduction to wavelets. Academic Press, Boston.

11. Akansu N, Serdijn WA, Selesnick IW (2010) Emerging applications of wavelets: A review. Phys Commun 3: 1-18.

12. Wei HT, Jeoti V (2004) A wavelet footprints-based compression scheme for ECG signals. Universiti Teknologi Petronas, Malaysia pp: 283-286.

13. Alfaouri M, Daqrouq K (2008) ECG signal denoising by wavelet transform thresholding. Am J Appl Sci 5: 276-281.
14. Raghuram M, Madhav KV, Krishna EH, Reddy K (2010) On the performance of wavelets in reducing motion artifacts from photoplethysmographic signals. In 2010 4th international conference on bioinformatics and biomedical engineering (iCBBE) pp: 1-4

15. Foo JYA (2006) Comparison of wavelet transformation and adaptive filtering in restoring artefact-induced time-related measurement. Biomed Signal Process Control 1: 93-98.

16. Comon P (1994) Independent component analysis: A new concept? Signal Process 36: 287-314.

17. Lee TW (1998) Independent component analysis: Theory and applications Boston: Kluwer Academic Publishers.

18. Hyvarinen A, Karhunen J, Oja E (2001) Independent component analysis. J Wiley, New York.

19. Kim BS, Yoo SK (2006) Motion artifact reduction in photoplethysmography using independent component analysis. IEEE Trans Biomed Eng 53: 566-568

20. Foo JY (2008) Use of independent component analysis to reduce motion artifact in pulse transit time measurement. IEEE Signal Process Lett 15: 124-126.

21. Sameni $R$ (2008) Extraction of fetal cardiac signals from an array of maternal abdominal recordings. Sharif University of Technology, Tehran, Iran.

22. Thakor NV, Zhu YS (1991) Applications of adaptive filtering to ECG analysis: noise cancellation and arrhythmia detection. IEEE Trans Biomed Eng 38: 785794

23. Xue Q, Hu YH, Tompkins WJ (1992) Neural-network-based adaptive matched filtering for QRS detection. IEEE Trans Biomed Eng 39: 317-329.

24. Kiryu T, Kaneko H, Saitoh $Y$ (1994) Motion artifact elimination using fuzzy rule based adaptive nonlinear filter. IEICE Trans Fundam Electron Commun Comput Sci E77-A: 833-838. 